\title{
Multichannel Electrotactile Feedback with Spatial and Mixed Coding for Closed-Loop Control of Grasping Force in Hand Prostheses
}

\author{
Strahinja Dosen, Member, IEEE, Marko Markovic, Matija Strbac, Minja Perović, Vladimir Kojić, \\ Goran Bijelić, Thierry Keller, Dario Farina, Senior Member, IEEE
}

\begin{abstract}
Providing somatosensory feedback to the user of a myoelectric prosthesis is an important goal since it can improve the utility as well as facilitate the embodiment of the assistive system. Most often, the grasping force was selected as the feedback variable and communicated through one or more individual single channel stimulation units (e.g., electrodes, vibration motors). In the present study, an integrated, compact, multichannel solution comprising an array electrode and a programmable stimulator was presented. Two coding schemes (15 levels), spatial and mixed (spatial and frequency) modulation, were tested in able-bodied subjects, psychometrically and in force control with routine grasping and force tracking using real and simulated prosthesis. The results demonstrated that mixed and spatial coding, although substantially different in psychometric tests, resulted in a similar performance during both force control tasks. Furthermore, the ideal, visual feedback was not better than the tactile feedback in routine grasping. To explain the observed results, a conceptual model was proposed emphasizing that the performance depends on multiple factors, including feedback uncertainty, nature of the task and the reliability of the feedforward control. The study outcomes, specific conclusions and the general model, are relevant for the design of closed-loop myoelectric prostheses utilizing tactile feedback.
\end{abstract}

Index Terms - Sensory feedback, force feedback, electrotactile stimulation, array electrodes, routine grasping, force tracking.

\section{INTRODUCTION}

$\mathrm{T}$ HE human hand is an extremely versatile effector capable of grasping a variety of objects reliably and consistently. It is also a sophisticated sensing instrument equipped with a dense network of tactile receptors providing high-fidelity

Submitted for review on July 31, 2015. This work was supported by the German Ministry for Education and Research (BMBF) via the Bernstein Focus Neurotechnology (BFNT) Göttingen under the grants No. 01GQ0817 and 01GQ0810, and the European Commission under the MYOSENS (FP7PEOPLE-2011-IAPP-286208) projects.

S. Dosen and D. Farina is with the Department of Neurorehabilitation Engineering, University Medical Center Göttingen (UMG), Georg-August University, Göttingen, Germany (e-mail: \{strahinja.dosen, dario.farina\}@bccn.uni-goettingen.de).

M. Markovic is with the Otto Bock HealthCare GmbH, Duderstadt, 37115, Germany (e-mail: marko.markovic@ottobock.de).

M. Strbac, M. Perović, V. Kojić, G. Bijelic, and T. Keller are with the Tecnalia Research \& Innovation, San Sebastian, Spain (e-mail: \{matija.strbac, minja.perovic, vladimir.kojic, goran.bjelic, thierry.keller\}@ tecnalia.com). information for the haptic exploration of the environment [1]. Amputation of the limb is an extremely traumatic event, leading to an irrevocable loss of these essential motor and sensory functions. A myoelectric prosthesis can be used to replace the missing hand. In these systems, the control is implemented by capturing the electrical activity of the remnant muscles to infer user volition and to control the prosthesis accordingly [2]. This approach can successfully replace the basic motor functions, with the available devices ranging from single degree of freedom (DOF) gripers to dexterous robotic hands [3]. However, with the current systems, the restoration is only partial since none of the devices, apart from one recent example [4], transmits the somatosensory information.

Transmitting the information about the current state of the prosthesis to the user is a long standing research challenge [5]. To this aim, the prosthesis is equipped with sensors, from which the data are acquired, suitably coded and communicated to the user by activating spared tactile sensory structures using invasive (e.g., direct nerve [6] or brain stimulation [7]) and non-invasive interfaces (e.g., surface electrocutaneous and mechanical stimulation [8], [9]). The skin sites to deliver intuitive feedback through tactile stimulation can be artificially created through a surgical procedure, known as targeted sensory reinnervation [10], which was developed following the success of targeted muscle reinnervation [11]. Both techniques are especially relevant for high-level amputations [12].

In surface electrotactile stimulation [8], the electrical current pulses are delivered to the skin through surface electrodes. The pulses depolarize the skin afferents and thereby create tactile sensations. The electrotactile feedback information can be transmitted by modulating the quality and intensity of the elicited sensations, i.e., by changing the stimulation parameters (pulse width, amplitude, and frequency coding) and/or location of the stimulation (spatial coding). To implement the latter, a multichannel interface is required. Contrary to the vibrotactile technology (e.g., pager motors), the stimulation parameters are fully decoupled and can be independently modulated. Since there are no moving mechanical parts, the electrotactile interface is also fast, compact and requires low power. Therefore, this technology is especially convenient for the implementation of high- 
resolution multichannel interfaces, which allow for flexible information coding by combining spatial and parameter modulation. However, a disadvantage of the electrotactile stimulation is that the elicited sensations can become uncomfortable at high intensities, limiting the dynamic range. Also, the electrical stimulation represents a strong interference to the recording of the myoelectric signals. These factors need to be considered for practical applications, e.g., the parameters should remain below discomfort threshold and the methods to avoid [13], [14] and/or suppress [15] stimulation artefacts need to be implemented.

The most obvious variable to feedback to the user is the grasping force, since it cannot be directly assessed through vision and it critically determines the grasp outcome. Indeed, most closed-loop hand prosthetic systems described in the literature provide feedback on grasping force [16]. Importantly, the feedback was provided using simple interfaces and coding schemes, i.e., a single stimulator transmitting the level of force through intensity and/or frequency modulation. The compact coin-type vibration motor commonly used in mobile phones was employed in [17], [18]. The motor has a single input that allows simultaneous scaling of the frequency and intensity of vibration (coupled parameters) proportionally to the detected grasping force. A combination of coin-type motors in a single housing can be used to generate more complex vibration patterns at the expense of the increased size of the interface [19]. The C2 tactor is a more sophisticated stimulator, which permits somewhat independent control of the vibration frequency and amplitude, however, with strong resonant effects [20], [21]. In [22], the duration and rate of vibration bursts at the base frequency of $200 \mathrm{~Hz}$ was used to transmit the grasping force information, whereas in [23] a simple amplitude modulation was employed. Single channel electrotactile stimulation with intensity and frequency coding was applied in [24] and [25], respectively.

Multichannel interfaces were considered as well, albeit less often. These were not dedicated, compact designs; instead, the multiple channels were obtained by combining several singlechannel stimulation units (electrodes or motors). An array of coin-type vibration motors [26], [27] or stimulation electrodes [27] placed along or circumferentially around the forearm was used to spatially code the level of grasping force [26] and hand aperture [27]. A technical solution for a four electrode interface relying on mixed coding to transmit 16 levels of grasping force (4 electrodes $\mathrm{x} 4$ pulse rates) was presented in [24]. However, the interface was not evaluated experimentally. Finally, one or more single-channel units exerting pressure on the skin, implementing modality matched feedback (force-to-force), were also developed and tested [28], [29]. The latter methods can provide more intuitive feedback to the user, compared to sensory substitution systems based on electro- and vibrotactile stimulation, but are also more cumbersome and difficult to implement and integrate into the prosthetic socket [16].

In the current study, a novel fully-integrated multichannel electrotactile interface is described and used to feedback the grasping force of an advanced myoelectric prosthesis. The force information was transmitted using two coding schemes to demonstrate the flexibility of the developed interface and assess the impact of the coding onto the closed-loop prosthesis control. The coding methods were devised to be identical in the number of levels (resolution) and yet lead to a substantially different psychometric performance. The resolution was determined by the physical maximum (number of channels) of the interface as well as spatial constraints of the forearm (circumference). The quality of force control for each coding method was evaluated using routine grasping and force tracking tasks. The results demonstrated that, contrary to initial expectations, the psychometric properties of the coding schemes did not translate to significantly different control performance. This outcome provides important insights into general principles of closed-loop control using tactile feedback. Furthermore, it implies that there is a flexibility when designing a feedback interface. Since the performance is similar, the coding can be selected based on other factors, such as, habituation to stimulation (diversifying patterns) or technical complexity (modulating a single vs. multiple parameters).

\section{METHODS}

\section{A. Feedback interface and coding schemes}

The system for electrotactile feedback comprised a programmable multichannel stimulation unit (MaxSens, Tecnalia, ES) and a flexible multi-pad electrode shown in Fig. 1(a). The stimulator generated biphasic, charge-compensated current pulses and the stimulation parameters could be adjusted online by sending simple textual commands over a Bluetooth connection. For the purpose of the present study, a custom-made communication and control protocol was implemented in the stimulator firmware, automatically mapping the prosthesis force value sent by the host PC to the stimulation parameters according to the selected coding method. Two coding schemes were implemented in the present study (Fig. 1), one using spatial (SPA) and the other using mixed (MIX) spatial and frequency modulation (details provided below). The prosthesis force was measured using an embedded force sensor by a prosthesis controller and sent to the host PC via a separate Bluetooth connection (see section II.B). The firmware also included a graphical user interface shown at the LCD, with the current stimulation parameters and the drawing of the electrode, similar to Fig. 1(b) and (c), with the color-coded channel states (on/off). The display was assessed by the experimenter during the pilot tests in order to ensure that the electrotactile coding was properly implemented.

The flexible electrode, designed to be placed around the circumference of the forearm, was $196.8 \mathrm{~mm}$ long and integrated 16 circular pads with a radius of $10 \mathrm{~mm}$ and centerto-center distance of $12.1 \mathrm{~mm}$. The distance between the electrode centers was selected considering the spatial discrimination threshold of the electrotactile stimulation as reported in [30] ( $9 \mathrm{~mm}$ on the forearm). The electrode 


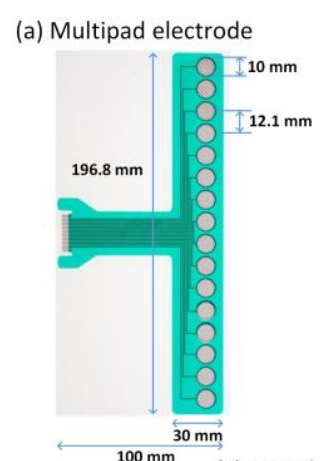

(b) Spatial coding (SPA)
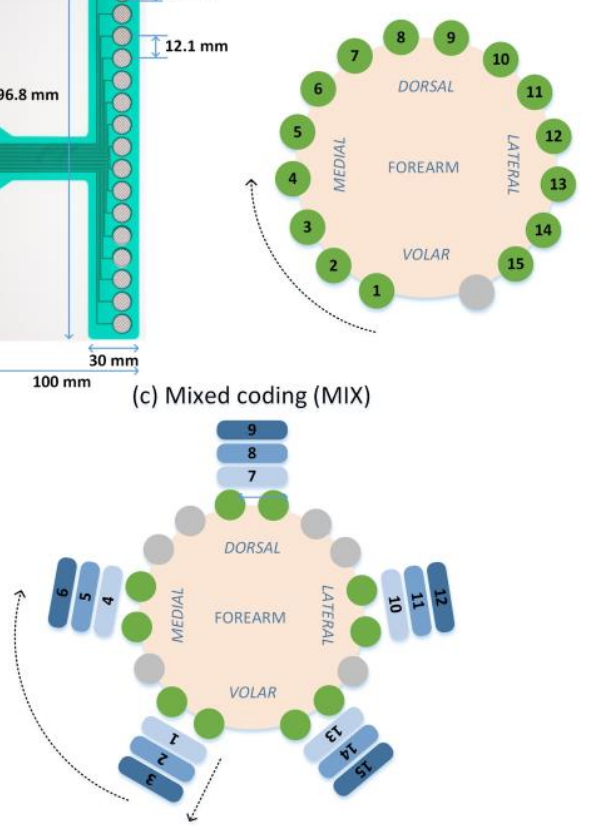

Fig. 1. The flexible and compact multi-pad electrode (a) and the two coding schemes, spatial (SPA) (b) and mixed (MIX) (c), used to transmit the grasping force information. The electrode was placed circumferentially around the subject forearm. In spatial coding, each pad corresponded to one level of grasping force. In mixed coding, the level was determined by a combination of the two activated pads and the stimulation frequency. In (b) and (c), the green circles are the electrode pads and the shades of blue are the frequencies, where darker color corresponds to the higher frequency. The numbers in (b) and (c) indicate the correspondence between the tactile codes (pad in SPA, two pads and frequency in MIX) and force levels (1 to 15).

comprised the base $150 \mu \mathrm{m}$-thick polyester layer with the imprinted conductive $\mathrm{Ag} / \mathrm{AgCl}$ traces and an insulation layer exposing the conductive circular pads. To improve the skinelectrode contact, the pads were covered by identically sized and shaped pieces of conductive hydrogel (AG702, Axelgaard, DK). The pads were used as the cathodes while a separate, standard stimulation electrode (PALS Foam, 5 x $9 \mathrm{~cm}$, Axelgaard, DK) acted as the common anode. For the present study, two electrodes were produced with the length of approximately 200 and $300 \mathrm{~mm}$, but only the shorter electrode was used since the other was too long for the subjects tested (i.e., the pads at the two ends would overlap). In some subjects the shorter electrode did not cover the full circumference of the forearm. For practical applications and optimal performance, the electrode should be produced in several sizes or custom-made (dimensioned) for the specific subject.

Two schemes for delivering the feedback information through the electrotactile interface were implemented, one using spatial coding (SPA) and the other exploiting the mixed spatial and frequency coding (MIX). The full range of the prosthesis grasping force $(0-100 \%)$ was divided into 15 equal subranges, where $100 \%$ corresponds to the maximum force of the prosthesis. In SPA, each electrode pad was associated to a single subrange. The electrode comprised 16 pads, and therefore the last pad was not used [Fig. 1(b)]. The stimulation at a specific pad indicated to the subject that the grasping force was currently within the subrange corresponding to that pad.
Therefore, the feedback was perceived by the subjects as the electrotactile stimuli moving along the circumference of the forearm, in the mediolateral direction when increasing and oppositely for the decrease in the feedback variable [Fig. 1(b)]. The spatial coding was selected since it has been used before as an intuitive approach to transmit the feedback information [26], [27]. Here, the subject needed to attend to a single stimulus property (location). The coding exploited all the available channels (1-15) except the last pad (16), which was not used since in some subjects the circumference of the forearm was too short to accommodate the full electrode. Since the electrode was designed considering the spatial discrimination threshold, we assumed that the subjects are likely to recognize the transition between the neighboring electrodes (relative discriminability). However, localizing precisely the ordinal number of the currently active channel (absolute discriminability), especially if the channel would be activated randomly (one out of 15), was expected to be a substantially more challenging task.

Therefore, a second scheme was designed based on the mixed coding. In MIX, the current subrange was denoted by a combination of the activated pad and the rate of pulse delivery [Fig. 1(c)]. Specifically, 5 groups of 2 neighboring pads were selected, and each pair of pads could be activated at three stimulation frequencies $(5,20$ and $50 \mathrm{~Hz})$, thereby coding 15 subranges of the prosthesis grasping force (5 pairs of pads $\times 3$ frequencies). Therefore, the feedback was felt by the subject as the electrotactile stimuli moving along the forearm and changing in frequency, in mediolateral direction between the pads and from lower to higher frequency within the pad for increase and oppositely for the decrease in the grasping force [Fig. 1(c)]. With MIX, the goal was to achieve the same overall resolution (15 subranges) as with SPA but with an improved absolute discriminability between the codes. To this aim, mixed coding allowed decreasing the number of spatial locations that needed to be recognized by the subjects (5 vs. 15), making them also more distinct as the locations were separated by the unused pads. However, the subjects in this case needed to attend to two different stimulus properties, i.e., location and frequency. Pads were activated in pairs to stimulate a larger area of the skin, improving perception and thereby closed-loop control, as demonstrated in [31]. For frequency modulation, the number of levels and distance in $\mathrm{Hz}$ ( $\Delta$ frequency) was selected so that the stimulation delivered at these frequencies to the same spatial location can be readily discriminated by the subjects (pilot tests).

\section{B. Experimental setup}

The experimental setup comprised (Fig. 2): advanced hand prosthesis (Michelangelo hand, Otto Bock Healthcare GmbH, AT), a multichannel EMG amplifier (INTEMG, OT Bioelettronica, IT), a multichannel electrotactile feedback interface (MaxSens, Tecnalia, ES) and a standard desktop PC with a 22 " monitor.

The Michelangelo prosthetic hand implements lateral and palmar grasps, where only the latter was used in the current study. The hand is instrumented with embedded sensors 


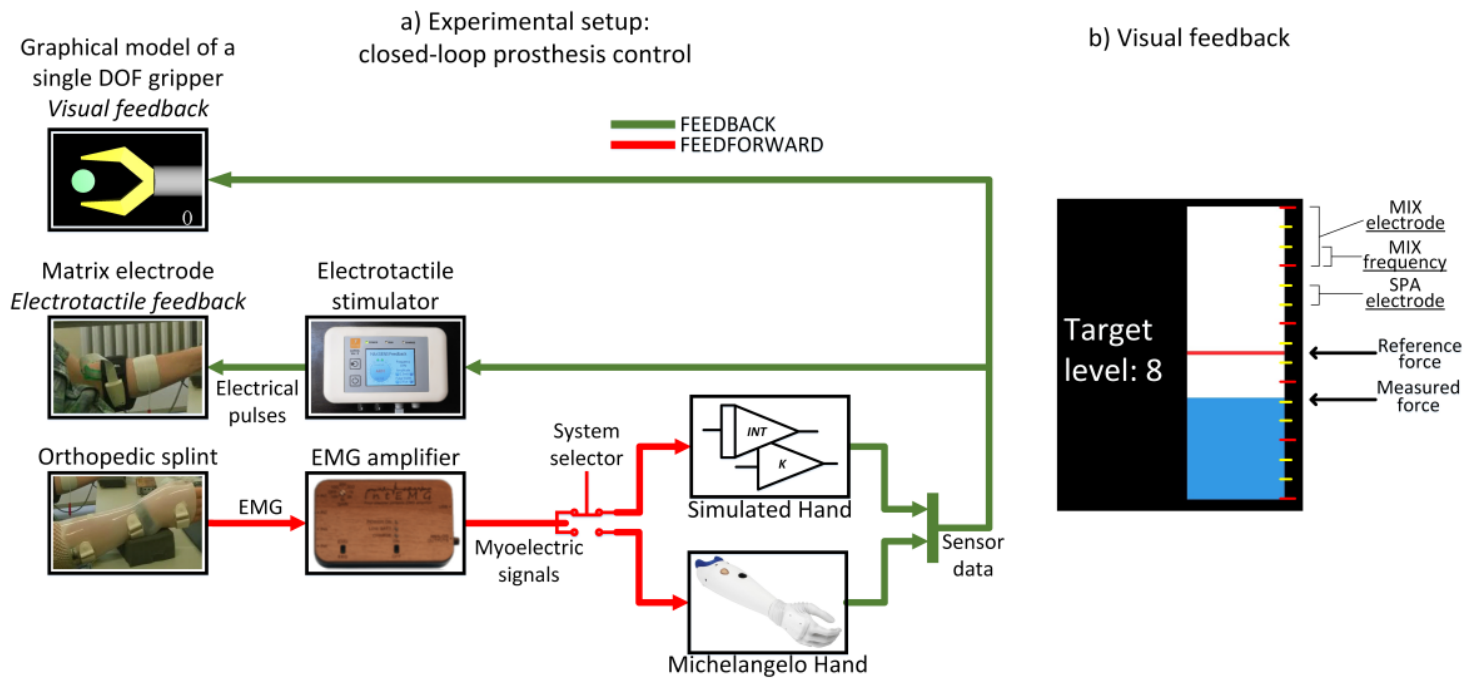

Fig. 2. Experimental setup. A system (a) for closed-loop control of grasping force using myoelectric interface to control a state-of-the-art hand prosthesis or its simulated model (virtual hand), and a multi-pad electrotactile interface to provide the grasping force feedback. To assess the prosthesis movement, the subjects observed a graphical model of a simple gripper displayed on the computer screen. In addition to the gripper, a force bar (b) was shown on the same screen just next to the gripper. The bar included the reference force level (red line) to be generated/tracked by the subject, currently measured force (blue bar, available only in VIS condition), and the horizontal ticks indicating the electrotactile coding. The latter was used to assist the subject in mapping the reference (desired) force to the desired electrotactile activity, i.e., active pad (SPA) or active pad and frequency (MIX). MIX and SPA stand for mixed and spatial coding, respectively.

measuring the motor position and grasping force (maximum $\sim 100 \mathrm{~N})$. It was connected to the desktop PC via Bluetooth, which was used to send commands to the hand and receive the sensor data sampled at $100 \mathrm{~Hz}$ by the internal hand controller.

The EMG amplifier included four bipolar channels, of which two were used in the current study. The amplifier sampled the EMG signals at $1 \mathrm{kHz}$ internally, segmented the data in $250-\mathrm{ms}$ windows with the $80 \%$ of overlap, calculated the mean absolute value (MAV) and sent it to the desktop PC via a USB connection. Standard $\mathrm{Ag} / \mathrm{AgCl}$ electrodes (Neuroline 720, AMBU, US) were placed over the hand and wrist flexor and extensor muscles in bipolar configuration. Before placing the electrodes, the skin was prepared by applying a small amount of abrasive gel (everi, Spes Medica, IT). To implement isometric contractions, the wrist and the metacarpophalangeal joints were immobilized using an orthopedic splint.

The multipad electrode for electrotactile feedback was placed on the subject forearm contralateral to the EMG electrodes to avoid the interference between the stimulation and EMG recording. The placement and calibration were performed as explained in the next section (II.C). Desktop PC ran an online control loop at $100 \mathrm{~Hz}$ implemented using a flexible test bench for prototyping and assessment of the human manual control systems [31], executing in real time through the Real Time Windows Target toolbox in Matlab Simulink. The test bench acquired the EMG signals and prosthesis sensor data, and based on this, determined and sent the commands to the prosthesis (myoelectric feedforward) and the measured grasping force to the stimulator (feedback). The stimulator implemented one of the two preconfigured electrotactile coding schemes (MIX and SPA), as explained in the previous section. The subjects proportionally controlled the velocity of the prosthesis opening/closing and grasping force; to close/open the prosthesis and increase/decrease the grasping force, the subjects activated the flexor/extensor muscles. The myoelectric signal gains were adjusted so that the maximal command was attained for approximately $70 \%$ of the maximum voluntary contraction, which was measured beforehand. A software switch was used to select the system to be controlled, i.e., a real prosthesis or a simulated model. The latter was implemented as a simple state machine emulating the prosthesis using an integrator or a pure gain depending on whether the prosthesis freely moves (closing/opening) or stalls (object contact), respectively. Therefore, during closing the myoelectric input set the prosthesis velocity, which was integrated to obtain aperture. After contact, the aperture remained constant, while the myoelectric input was multiplied by a gain to set the grasping force. This reflects the operation of most of the myoelectric prostheses, and the model parameters have been adjusted using experimental data to emulate the operation of the Michelangelo Hand.

The subject was comfortably seated in a chair in front of a desk. To eliminate the uncontrolled sources of feedback (e.g., motor vibrations), the prosthesis was detached from the subject, and placed on a separate table, out of his/her sight. Also, the subject wore noise-canceling headphones during the tests. An object was positioned between the prosthesis fingers so that when the prosthesis closed, it contacted the object. The subject looked at the monitor, which displayed a graphical model of a simple gripper replicating the movement of the real and simulated prosthesis and grasping a stiff virtual object. The gripper was a simple geometrical model, and therefore very different from the real prosthesis, but the maximum aperture measured on the screen was approximately the same as in the real prosthesis $(\sim 11 \mathrm{~cm})$. Similarly, the diameter of the virtual object was set to correspond to the aperture of the 


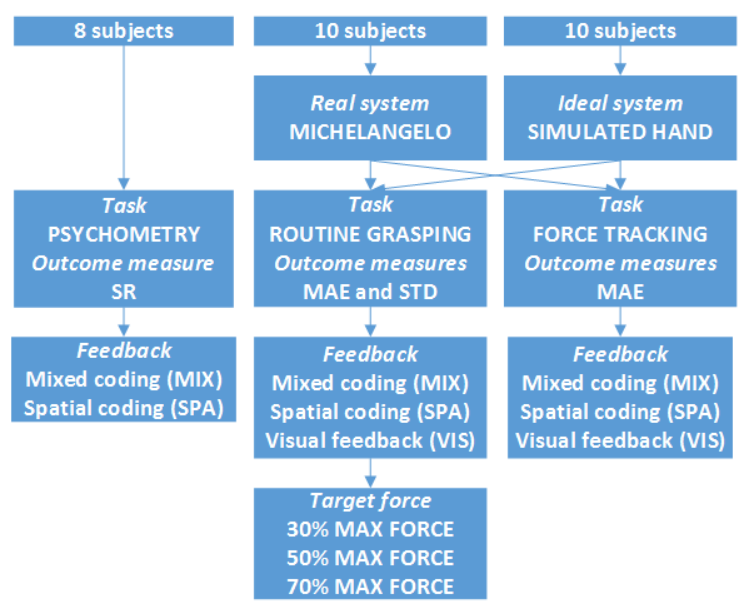

Fig. 3. Experimental protocol. Three groups of subjects were tested in a psychometric test and closed-loop force control with real and simulated prosthesis, respectively. The closed-loop force control included two tasks (routine grasping and force tracking) and each task was performed in three feedback conditions, namely, electrotactile stimulation with mixed (MIX) and spatial (SPA) coding and visual feedback (VIS). Finally, for the routine grasping, each feedback condition was tested with three target forces $(30,50$ and $70 \%$ of the maximum force). The outcome measures were success rate (SR) in the psychometric test, mean absolute error (MAE) with respect to the target level and its standard deviation (STD) in the routine grasping, and mean absolute error (MAE) with respect to the reference trajectory for the force tracking.

hand when closed $(\sim 2 \mathrm{~cm})$. Depending on the test condition (see section II.D), a visual feedback of grasping force was provided to the subject by displaying a vertical bar, positioned next to the prosthesis. The vertical bar spanned $80 \%$ of the 22 " screen, mapping the full range of forces $(0-100 \%)$, with the subject approximately $50 \mathrm{~cm}$ away from the display. The structure of the experimental protocol is explained in the next two sections and depicted in Fig. 3.

\section{Experimental protocol: Psychometric evaluation}

To evaluate how reliably the subjects can recognize the state of the feedback variable using the two coding schemes (MIX and SPA), a psychometric evaluation was conducted. Eight able-bodied volunteers ( $31 \pm 6$ yrs.) signed an informed consent form for the test approved by a local ethical committee. Both coding schemes were evaluated in a random order in each subject. The multipad electrode was placed around the forearm at the distance of approximately $1 / 3$ of the forearm length proximal to the elbow, so that the middle pads located next to the electrode connector [Fig. 1(a)] were positioned in the middle of the dorsal side of the forearm. The subjects were comfortably seated, resting the forearm on the table in front of them. First, the sensation thresholds (ST) were determined for each electrode pad using the method of limits [32]. The stimulation frequency was set to $50 \mathrm{~Hz}$, pulse width to $300 \mu \mathrm{s}$ and the current amplitude was increased in steps of $0.1 \mathrm{~mA}$ until the subject reported that he/she felt the stimulation. The current amplitude for the pad was set to 1.2.ST and kept constant during the experiment, ensuring comfortable and well-localized sensations.

The psychometric test comprised three phases: familiarization, reinforced learning and validation. The first two phases were used as training and the last phase for performance assessment and data collection. In the familiarization phase, all 15 tactile stimuli coding the levels of the feedback variable (grasping force) were presented to the subject, from the lowest to the highest, so that the subjects experienced the full set of possible tactile stimuli. In the reinforced learning phase the levels were randomly presented by delivering the stimulation at a specific electrode pad (SPA) or at a specific pad group and frequency (MIX) for 1 second, after which the subjects were asked to guess the presented level, by reporting a number from 1 to 15 . The experimenter then provided verbal feedback about the correct answer, by saying "correct" if the subject successfully guessed the level or by saying "incorrect" and reporting the correct number (115). Each level was presented 3 times in total $(3 \times 15=45$ trials). Finally, the same protocol was repeated in the validation phase, except that each level was delivered 7 times (105 trials in total) and the subjects did not receive the verbal feedback about the correct answer. In all the tests, the pause between the two successive stimuli was 5 seconds. Short rest breaks were made on subject request. The total time for the training (familiarization and reinforced learning phase) was approximately $30 \mathrm{~min}$, while the validation phase took between $45 \mathrm{~min}$ and $1 \mathrm{~h}$.

\section{Experimental protocol: Routine grasping and force tracking}

The closed-loop control of grasping force was evaluated using force tracking and routine grasping tasks. Each task was performed using visual (VIS) and electrotactile feedback with two coding schemes (SPA and MIX). The aim of VIS condition was to assess the control with an ideal feedback interface (benchmark performance). SPA and MIX coded the grasping force with the resolution of 15 equidistant segments, as explained in section II.A. Two groups of able-bodied subjects were recruited, one group (10 subjects, $29 \pm 5$ yrs.) performed the tests with the real prosthesis and the other (10 subjects, $33 \pm 8$ yrs.) using a simulation of the prosthesis (ideal system). The simulated hand was included in order to evaluate the effect of the physical properties (e.g., friction of the mechanism, inertia and time delays) on the closed-loop control.

First, the subjects received a short training $(5-10 \mathrm{~min})$ in myoelectric signal generation and control (prosthesis disconnected) during which they were asked to modulate the myoelectric signals according to the reference trapezoid profiles shown on the screen. The principle of operation of the myoelectric prosthesis as well as the electrotactile feedback was then explained. The feedback was demonstrated by iterating through the full range of force levels (1-15) while simultaneously showing the force on the screen [visual bar, Fig. 2(b)] and delivering the corresponding electrotactile stimulus. The force level was modulated continuously, and the bar was segmented in 15 levels, equivalently to the resolution of the tactile feedback [Fig. 2(b)]. Therefore, the subject could relate a transition between the electrodes (SPA) or between the electrodes and pulse rates (MIX) to the transitions of the force 
level between the segments on the bar. This was repeated for up to five full cycles (from 1 to 15), or until the subject reported that she/he perceived and understood the electrotactile feedback. Finally, up to five additional cycles were performed but this time without the visual feedback (bar removed, Fig. 2(b)). Therefore, the subjects had to guess the current force by recognizing the electrotactile stimulus. They reported verbally the force level that they perceived from the stimulation, as a number from 1 to 15 , and received the feedback from the experimenter about the correct answer (reinforced learning). If the subject guessed all the forces correctly, the training was stopped. The feedback training lasted less than 15 min.

The routine grasping task evaluated the subject ability to grasp objects in a straightforward manner, by increasing and then holding the contraction level (feedforward command) so that the grasping force upon initial contact with the object was at the desired force level [33], [34]. The reference forces were set to 30,50 and $70 \%$ of prosthesis maximum and for each level the subjects performed 70 trials. The first 30 trials were considered as the training phase and were not taken into account in the data analysis (see section II.E). The task for the subjects was to close the hand from the fully open position and grip the object so that the resulting grasping force was as close as possible to the desired one. After the hand grasped the object, the subject relaxed his muscles, indicating the end of the trial. Since the prosthesis (real and simulated) was nonbackdrivable, it would maintain the attained force and then open automatically after $1 \mathrm{~s}$. Therefore, the subjects did not need to open the hand manually. Hand opening was anyway irrelevant for the task and the subjects could rather focus on closing and producing correct forces. The subjects were instructed to use the feedback about the grasping force achieved in the current trial as error information to update the feedforward command in the next trial. More specifically, when the subject grasped the object, electrotactile feedback started transmitting the grasping force. Due to the nature of the routine grasping task, the force jumped abruptly to the level corresponding to the user EMG command. The force than remained constant, and the feedback was transmitting the code corresponding to that force, until the hand auto-opened. The subject had to interpret this code in order to determine the level of grasping force attained in the present trial. By comparing the generated and desired force, the subject updated the EMG command in the next trial. Before starting the sequence of grasping trials, the desired level of force to be generated was verbally indicated to the subject and shown on the screen using a reference bar [Fig. 2(b)]. In the conditions with the electrotactile feedback, the desired outcome was also indicated by briefly activating (10 s) the electrode (SPA) or electrode and frequency (MIX) corresponding to the reference force.

The force tracking task evaluated the subject's ability to modulate the grasping force while the hand was closed around an object. This skill is relevant whenever there is the need to change the strength of the grasp in a controlled manner, for example, carefully releasing or gripping a delicate object, or handing over an object from hand to hand or to another person. From the fully open state, the prosthesis automatically closed around the object. This function was implemented in the main control loop on the host PC for both simulated and real prosthesis. When triggered by the experimenter, the function would send commands to the prosthesis to implement a predefined velocity profile: the hand started closing faster and then slowed down to contact the object gently and generate a low baseline force of approximately 5\%. From this point, the subject took over the control and the task was to modulate the grasping force so that it tracked a reference trajectory (trapezoidal profile): for $3 \mathrm{~s}$ force remained at the baseline, during $15 \mathrm{~s}$ it linearly increased to $87.5 \%$ ( $14^{\text {th }}$ level), stayed at the plateau level lasting $3 \mathrm{~s}$, and finally during $15 \mathrm{~s}$ the force linearly decreased back to the baseline. In VIS, both the reference (red line) and the measured force (blue bar) were shown [Fig. 2(b)], while in SPA and MIX only the reference line was available (blue bar hidden). The reference trajectory was presented two times per trial, and each subject performed three trials in each feedback condition. The first trial was regarded as the training and was not used for data analysis.

\section{E. Data analysis}

The main outcome measure in the psychometric test was the percent success rate (SR). In MIX, the recognition was deemed successful if the subject correctly guessed both the location and frequency of stimulation. The statistically significant differences between SPA and MIX were assessed using a paired t-test. In the routine grasping task, mean absolute error (MAE) from the desired force and the standard deviation (STD) were calculated per subject to evaluate the accuracy and precision in force control, respectively. To assess the quality of force tracking, MAE was computed between the generated and reference force profiles. The MAE in this case represented the tracking error averaged over the samples of the force signal acquired at $100 \mathrm{~Hz}$. To compare the conditions, a mixed-model ANOVA was applied, with the HAND (VIRT or REAL) as between-subject factor, and FEEDBACK (VIS, SPA and MIX) and force LEVEL (30, 50 and $70 \%$ ) as within-subject factors. Tukey's honestly significant criterion (HSD) was used for the pairwise comparison. The threshold for the statistical significance was set to $p<0.05$.

\section{RESULTS}

\section{A. Psychometric tests}

The subjects were significantly better in discriminating electrotactile stimuli when using MIX compared to SPA. The average SR (mean \pm standard deviation) was $36 \pm 11 \%$ for SPA and $87 \pm 10 \%$ for MIX ( $p<0.001)$. The confusion matrices are depicted in Fig. 4, and they exhibit clear structural patterns revealing the perceptual properties of the two coding interfaces. The diagonal line is sharper for MIX, indicating better recognition. The two parallel lines directly next to the main diagonal represent the cases in which the subjects correctly guessed the active electrode, but misrecognized the 


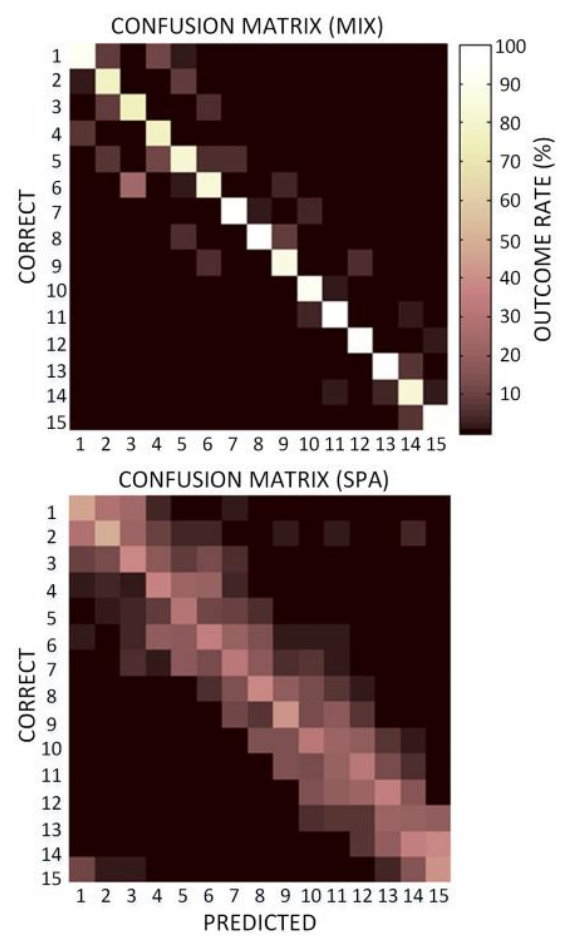

Fig. 4. The confusion matrices for the mixed (MIX, top) and spatial (SPA, bottom) coding. The success rates were significantly higher when using MIX. The numbering of the correct and predicted classes (levels) corresponds to the numbering in Fig. 1.

frequency. The error was limited to the neighboring frequency. The two parallel lines separated by a one-line gap with respect to the main diagonal correspond to the cases in which the subjects misclassified the active electrode and correctly guessed the frequency (in all but two cases). This error was also limited to the direct neighbors of the activated pad group. On the other side, the confusion matrix for SPA is characterized with a blurred area around the main diagonal, spanning two to three fields off the main diagonal consistently across all the classes. The subjects' answers were therefore distributed within several levels around the correct stimulus. The recognition for SPA was within one-level off the correct class in $70 \pm 9 \%$ cases, which is still significantly worse compared to the correct classification in MIX $(p<0.01)$.

\section{B. Routine grasping}

A representative example of the generated forces in the routine grasping task for a subject using Michelangelo prosthesis with spatially coded electrotactile feedback (SPA) is shown in Fig. 5. Across trials, the generated forces scattered around the target force level (dashed red line), with the variability increasing for the higher target forces. In terms of the generated force levels (active electrodes), the forces were confined to one level above or below the correct level when the target was $30 \%\left[5^{\text {th }} \pm 1\right.$ electrode, Fig. 5(a)]. For 50\% [Fig. 5 (b)], most of the forces were still in the same zone $\left(8^{\text {th }} \pm 1\right.$ electrode), but there were some forces further away (2-3 levels off the target). Finally, for the highest target [Fig. 5(c)], the forces varied within the band of 3 to 4 levels from the correct one $\left(11^{\text {th }}\right.$ electrode), with one case in which the force saturated at the maximum $(\sim 1)$. Nevertheless, the generated forces for all the target levels demonstrate that the subject successfully utilized the feedback to maintain the grasping force in the vicinity of the target during a sequence of repeated grasping trials. The force traces exhibit variability but there is no visible drift with respect to the target level, meaning that the subject constantly updated the feedforward command based on the error in the previous trial in an effort to stay close to the desired force.

The summary results for all the conditions in the routine grasping task are depicted in Fig. 6. The statistical analysis for the average MAE revealed that LEVEL was a significant factor $(p<0.001)$ and that there was a significant interaction between FEEDBACK and LEVEL $(p<0.05)$. The post hoc test however determined that there was no significant difference between FEEDBACK conditions within any of the LEVELs. Across LEVELs [Fig. 6(b)], the performance for

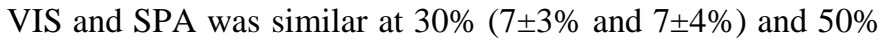
$(9 \pm 3 \%$ and $9 \pm 2 \%)$, and significantly worse at $70 \%(12 \pm 2 \%$ and $13 \pm 3 \%$ ), whereas for MIX the performance dropped significantly for each increase in the target force, from $6 \pm 2 \%$ for $30 \%$ to $11 \pm 4 \%$ for $50 \%$ and $14 \pm 4 \%$ for $70 \%$. The factor HAND was non-significant. Regarding the dispersion [Fig. $6(\mathrm{c})]$, the only significant factor was LEVEL $(p<0.001)$. The dispersion of the generated forces increased steadily and significantly with the higher target forces. The STD was $7 \pm 3 \%, 11 \pm 2 \%$ and $14 \pm 2 \%$ for the LEVEL of 30,50 and $70 \%$, respectively. Overall, the performance of the closed-loop prosthesis control, both in terms of precision and accuracy, deteriorated consistently for increasing target forces. Within each level, all three feedback types performed similarly, and this was regardless of the controlled system (virtual or real prosthesis). The box plots of the generated forces for all subjects across levels and conditions are shown in Fig. 6(a), demonstrating that the performance, in terms of median and dispersion, was indeed similar within the target force levels and deteriorated from lower to higher levels.

\section{Force tracking}

Figure 7 depicts reference (red dashed line) and generated (black continuous line) force trajectories for a subject performing a tracking task using visual [Fig. 7(a) and (c)] and electrotactile feedback with spatial coding [Fig. 7(b)]. In both cases, the generated trajectory comprised a sequence of abrupt changes in the grasping force (staircase profile). This nonsmooth force control is an intrinsic property of the prosthesis, and reflects the fact that the motor torque needs to overcome internal friction in the prosthesis mechanism. The variability of the surface myoelectric control signals also contributed to the stepwise response, by limiting the precision and reliability with which the subject could increase/decrease the control input into the prosthesis. Since the prosthesis is nonbackdrivable, the force is set to the maximum of the noisy myoelectric input (if it overcomes the internal friction, as explained before). With the visual feedback, the quality of tracking was better, with MAE of 5.2\% for VIS compared to MAE of $9.7 \%$ for SPA [Fig. 7(a) versus (b)]. The subject 


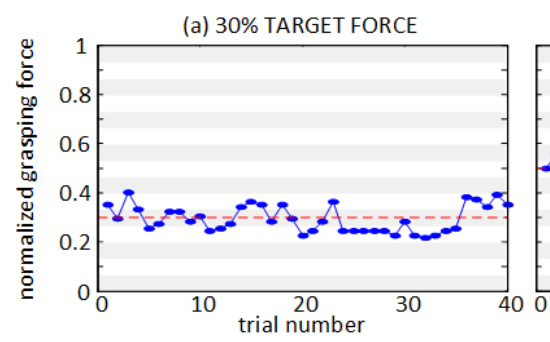

(b) 50\% TARGET FORCE

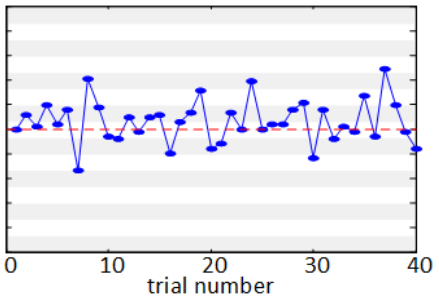

(c) 70\% TARGET FORCE

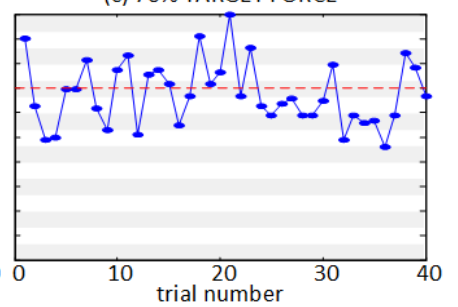

Fig. 5. Example sequences of the generated forces (blue dots) in the routine grasping task for a subject using Michelangelo prosthesis with electrotactile feedback and spatial coding (SPA) to generate the target force (red dashed line) of (a) 30\%, (b) 50\% and (c) $70 \%$ of the maximum prosthesis force. The grasping force in the plots is normalized to the prosthesis maximum. The subject relied on the feedback to maintain the forces in the vicinity of the target level with the dispersion increasing for the higher targets.
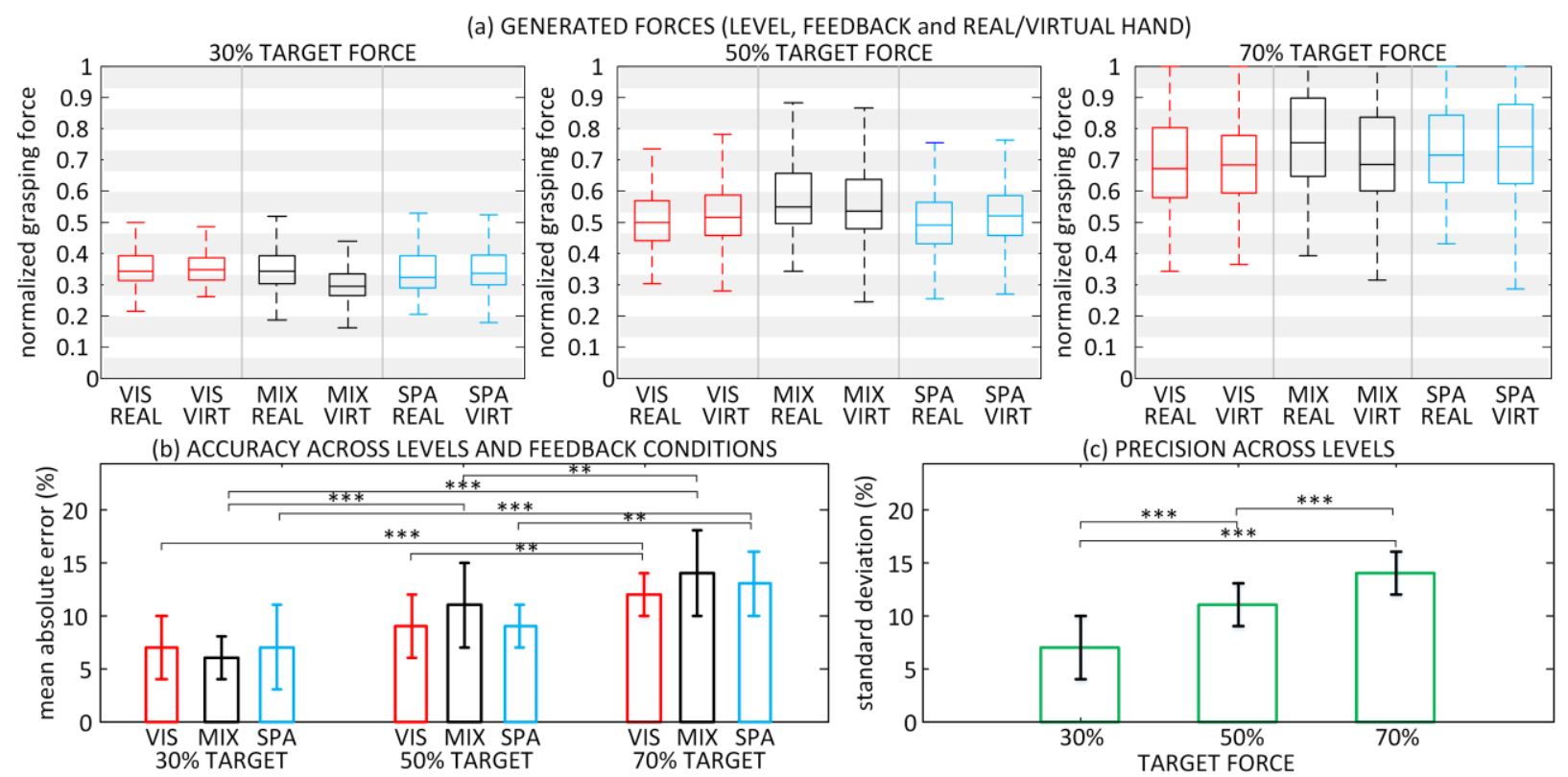

Fig. 6. The summary results for the routine grasping task: (a) box plots of the generated forces for all subjects, (b) accuracy across levels and feedback conditions averaged over the controlled system (non-significant factor), and (c) precision across levels averaged over the feedback type and controlled system (non-significant factors). The performance of the feedback conditions was similar within each target force level irrespective of the controlled system. Annotation: VIS, SPA and MIX - visual feedback, electrotactile spatial coding, electrotactile mixed coding, respectively; VIRT, REAL - simulated and real hand, respectively. The alternating white and gray strips in (a) represent the force levels (1-15) for the electrotactile coding. The horizontal bars indicate statistically significant differences $(* *, p<0.01 ; * * *, p<0.001)$.

made smaller steps during the force increase and decrease. Finally, the best tracking was obtained when the subject used visual feedback to control the simulated hand $(\mathrm{MAE}=4.1 \%)$, but the step changes in the force are still noticeable [Fig. 7(c)]. This effect was now solely due to the limitations of the surface myoelectric control, since the virtual hand eliminated the influence of the physical system parameters.

Summary results for the force tracking task are shown in Fig. 8. The FEEDBACK was the only significant factor $(p<$ $0.001)$. The average MAE for VIS was $7 \pm 2 \%$, and VIS outperformed the electrotactile conditions MIX and SPA $(p<$ $0.001)$. The performance of MIX $(15 \pm 4 \%)$ and SPA $(14 \pm 3 \%)$ was similar. The factor HAND as well as the interaction between the HAND and FEEDBACK were non-significant.

\section{DISCUSSION}

A compact and flexible interface for the delivery of electrotactile feedback through a multipad electrode was presented. Psychometric tests were conducted to assess the success rate in recognizing a set of 15 stimuli using spatial (15 locations) and mixed (5 locations $x 3$ frequencies) coding. The tests demonstrated that the spatial coding was difficult for the subjects, whereas the mixed coding was recognized with a high success rate. Therefore, the mixed coding can be used to reliably transmit high-resolution information (15 levels) after a brief training of less than $30 \mathrm{~min}$, and also allows for a more compact interface. These are potential advantages of the mixed coding compared to the pure spatial or parameter modulation schemes [17], [18], [26], [27]. The present study investigated the electrotactile stimulation, but equivalent coding schemes could be implemented using vibration motors. However, simple vibrators, such as pager motors, have a rather poor dynamic range and the vibration parameters, intensity and frequency, are not independent. Nevertheless, in the mixed coding scheme, the requirements are minimal, i.e., each vibrator needs to provide three distinct sensations (low, medium and high). As demonstrated in our previous study [35], the human subjects can achieve a reliable recognition of 


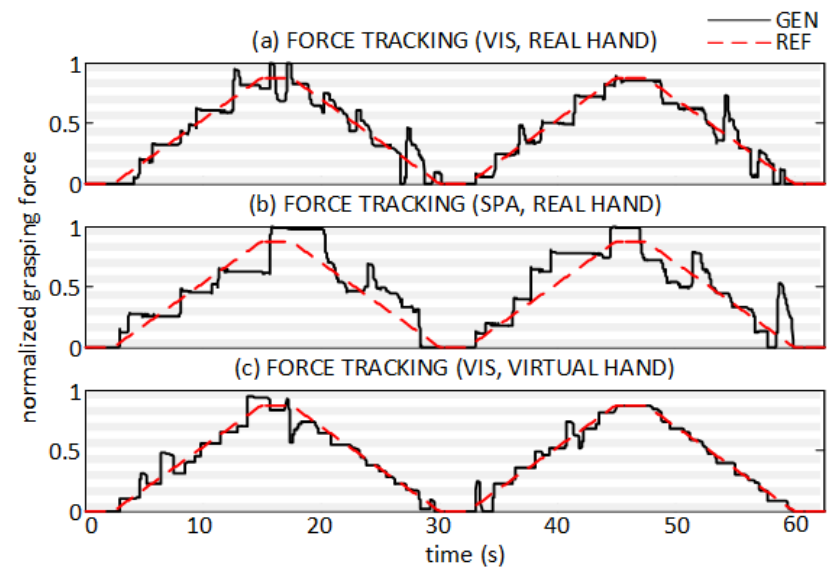

Fig. 7. Representative force tracking trials for a subject using visual feedback with (a) real hand and (c) virtual hand, and (b) electrotactile feedback with spatial coding and real hand. The generated trajectory (black continuous line) is stepwise, reflecting the nature of the prosthesis operation in combination with the variability of the myoelectric control signals. The best tracking was achieved using visual feedback and virtual hand. The alternating with and gray strips represent the force levels (1-15) for the electrotactile coding.

three levels (> 80\%), even with the simple motor. Finally, if necessary, more advanced solutions, such as $\mathrm{C} 2$ tactor, can be used.

The closed-loop prosthesis control tests demonstrated that, contrary to psychometric tests, the conclusions in this case are not as straightforward. Surprisingly, despite the substantial difference in the psychometric properties of the two tactile coding schemes, the performance with SPA and MIX was similar in both closed-loop control tasks. To explain this and the other results, we propose a conceptual, qualitative scheme given in Fig. 9. To understand and predict the performance of closed-loop control in prosthetics, the feedback needs to be considered as one (dependent) component of a more complex system. Therefore, knowing the characteristics of the feedback channel in isolation (e.g., psychometrics) is not enough to predict the outcome. The performance will ultimately depend on the feedforward interface as well as on the nature of the task. The performance depends on the uncertainty of the feedback interface. This is defined by the resolution (number of levels coded) and refers to the capability of the human subject to recognize the levels both absolutely (recognize a randomly selected stimulus) or relatively (recognize a transition between the two neighboring stimuli). The nature of the tasks for which the prosthesis is being used determines which of these dimensions are actually relevant for the task accomplishment. Finally, the uncertainty of the feedforward command pathway will ultimately affect the control capability [36]. Therefore, the complex interaction between these elements defines the effective resolution of the closed-loop system and consequently its overall performance.

This is a similar interaction to that characterizing normal human motor control. As demonstrated in motor adaptation experiments [37], the closed-loop behavior of the human subject, such as his/her adaption rate, depends on the uncertainty of both feedback and feedforward pathways. Furthermore, the humans are capable of sensor data fusion, state estimation and optimal and predictive control [38], [39],

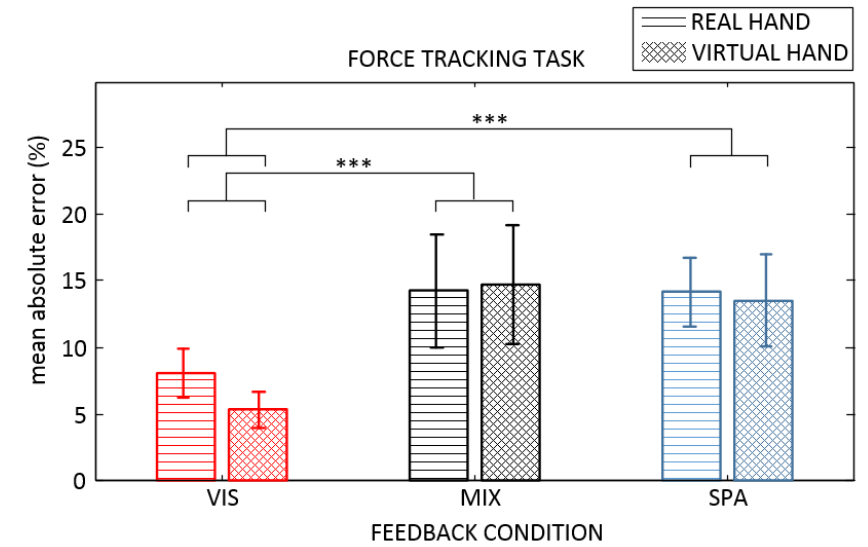

Fig. 8. Summary results for the force tracking task using simulated and real hand, visual feedback (VIS) and electrotactile feedback with spatial (SPA) and mixed coding (MIX). The horizontal bars indicate the statistically significant differences for the factor FEEDBACK $(* * *, p<0.001)$.

and these abilities are likely to fundamentally impact the performance of prosthetic control as well. The fact that basic principles of human motor control needs to be considered when designing closed-loop systems in prosthetics has been acknowledged in literature and the research is in progress [26], [34], [36].

The first important implication of these considerations is that the standard psychometric tests evaluating the absolute level recognition might not be enough to conclusively evaluate the performance of the feedback interfaces. Instead, the interfaces need to be evaluated in the context of a relevant control task. In the present study, the ability to correctly recognize a randomly presented stimulus, as measured by the psychometric assessment, was not really fundamental for accomplishing the task. The subjects could also rely on detecting the transitions between the neighboring pads across trials (routine grasping) or time (force tracking), indicating the direction and amplitude of the force change. Therefore, a disadvantage of SPA with respect to MIX could have been rendered less relevant by the nature of the task, more specifically, by the possibility to exploit the relative discrimination and control. As stated in section II.A, the electrode was dimensioned considering the spatial discrimination threshold, and therefore, the subjects were likely able to detect the transition of the stimulation between the two neighboring pads. This might have been applicable to both routine grasping and force tracking, and especially relevant for the latter one. However, the quality of relative discrimination was not explicitly assessed in the present study, and therefore these are only hypotheses (as also indicated in Fig. 9). The exact performance of MIX versus SPA in relative discrimination could be determined only through a dedicated test. Finally, the uncertainty of the feedforward control might have contributed in flattening out the potential advantage of MIX over SPA.

In the routine grasping task, the electrotactile feedback resulted in a similar performance with respect to both precision and accuracy of the generated forces compared to the benchmark visual feedback, and this was regardless of the controlled system (virtual or real) and the target force (low, 


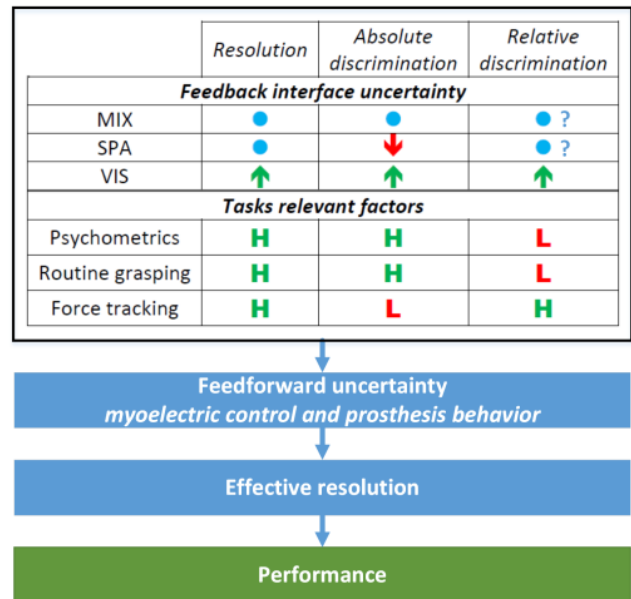

Fig. 9. The conceptual scheme depicting the model of the closed-loop prosthesis control. The uncertainty of the feedback interface interacts with the parameters relevant for the task, and after taking into account the reliability of the feedforward command pathway, determines the effective resolution of the closed-loop control. Annotations: $\uparrow, \bullet, \downarrow$ - the feedback interface has higher, equal or lower performance, respectively; H, L high and low importance for the task, respectively; ? - indicates an indirect hypothesis which was not explicitly tested.

medium and high). This is an encouraging outcome implying that a high-resolution electrotactile feedback, transmitted using the presented multipad interface, can still lead to the maximal (benchmark) performance across the full force range, despite the inevitable reduction in the quality of information transmission with respect to vision (i.e., discrete vs. continuous information, recognition errors). Importantly, these results were obtained by allowing the subjects to have a clear visual assessment of the prosthesis movement, making the setup and conclusions more practically relevant. Specifically, in all the conditions of the routine grasping task, the subjects were looking at the virtual gripper. Although the gripper represented a greatly simplified model of the prosthesis, such a feedback was provided so that the subjects could observe the hand aperture and estimate the closing velocity under standard conditions (i.e., same for all subjects). In real life, the users usually look at the prosthesis while grasping, and as demonstrated before [33], the closing velocity is an important input that can assist the subjects in force control across routine grasping trials. The fact that electrotactile feedback resulted in the similar performance as the benchmark visual feedback can be attributed to the uncertainty of the feedforward pathway [33], [34]. The myocontrol based on surface EMG signals is an inherently noisy command interface. Due to the inability to precisely control the generated command, the subjects could not translate the most precise information on the grasping force, provided using the visual interface, into the better control performance. This conclusion was confirmed by the fact that the performance across the feedback conditions remained similar also when using the simulated hand. Previous studies investigating the accuracy and precision of the prosthesis force control also recognized the impact of the feedforward channel on the closed-loop [33], [34]. The higher target force increased the dispersion and decreased the accuracy during repeated grasping and also affected the effectiveness of feedback [33]. Furthermore, the reliability of the feedforward interface (EMG vs. joystick) impacted on the quality of feedback-driven learning [34]. Finally, the difficulties in control were pointed out in [22] as one possible reason why vibrotactile feedback failed to improve the performance in naïve users.

Nevertheless, in the force-tracking task, the visual feedback did outperform the tactile stimulation. This implies that a specific nature of a concrete task needs to be considered when designing the closed-loop control system. In this case, the reference force was smoothly and gradually increased from the baseline to the maximum level. Visual feedback provided precise and continuous force information [Fig. 2(b)]. Therefore, the subject could react by increasing/decreasing the force as soon as the reference force (red line) surpassed the measured one (blue bar). Since the subjects monitored the movement of the reference, they could even employ the predictive control reacting in anticipation of the change. With the practical tactile feedback, the subjects could exploit the same mechanism, but with the greater uncertainty, due to limited resolution and difficulties in perception. Therefore, for this specific task, a reliable tactile interface with an even higher resolution (e.g., using more locations and/or frequency levels in MIX) might be beneficial for performance. In the routine grasping, however, the plateau performance has been already reached. However, this applies to the present setup, which included noisy command interface (surface EMG) and a prosthesis with a rough force modulation. Currently, there is an intensive research towards developing implanted myoelectric sensors [40], [41] enabling more accurate and precise command generation. As explained before, the control interface seemed to be the main limiting factor in the routine grasping task, and therefore, implanted solutions can allow the user to benefit from the higher resolution feedback and thereby improve the control performance. Finally, novel prosthetic devices might be developed allowing fine force control, such as a low-impedance prosthesis presented in [42], which in combination with the better control can also have an important impact on the utility of the feedback.

The prosthesis control loop operated in real time on the host PC. Nevertheless, there were inevitable delays when using the real prosthesis, since the commands and sensor data were transmitted via a Bluetooth connection with a delay of approximately $60 \mathrm{~ms}$ and $50 \mathrm{~ms}$, respectively. When using the simulated prosthesis, there were no such delays since the simulation ran at the host. Importantly, the loop delays did not play a role in the routine grasping task, since as explained before, it was performed using feedforward control, i.e., there were no feedback-driven corrections within an ongoing trial but only trial-by-trial adjustments. It seems to be the same for the tracking task, where controlling the simulated hand (with no communication delays) did not change the performance (Fig. 8).

The presented study demonstrated the effectiveness of a novel compact interface for the multichannel feedback in hand prostheses and also revealed some important insights relevant for its application as well as further development of prosthesis 
feedback systems. The interface as well as the whole control loop represent a flexible setup that can be used to test arbitrary coding configurations, such as, spatial coding with lower resolution but higher spatial separation (e.g., using every $N$ th pad), intensity and/or frequency modulation with more levels, or combinations (mixed coding). The present study is a first step in that direction, starting from the maximum that the interface could provide (15 levels) and elucidating general principles (Fig. 9) rather than specific solutions. An important future goal will be to use the developed test bench for a systematic investigation of coding configurations, in search for an optimal setup. For example, one could start from few levels and then increase the resolution of the feedback, level by level, each time testing the prosthesis control performance. It is likely that there will be a resolution at which the performance will reach a plateau, and this might be less than 15 levels used in the present study. After the resolution is determined, one can test an optimal coding scheme (spatial vs. parameter vs. mixed modulation) to transmit that number of levels. However, as implied by the present study, this could be a rather difficult quest since the solution might depend on the command interface (surface vs. implanted EMG recording) as well as the task (grasping or force modulation). At the end, the selected configuration might be a trade-off between technical complexity, relevant tasks and user experience.

An important step is the evaluation of the system in the functional tasks of daily living using standardized tests [43]. In the present study, a full force range was mapped linearly by the feedback. However, this might not be an optimal solution when considering daily-life tasks. For example, it might be more relevant for the user that the feedback provides higher resolution (smaller subranges) for low and medium forces compared to high forces, in order to facilitate manipulation of delicate objects. Therefore, the best mapping from the force range to the feedback codes is yet to be determined. This is also related to the available force range, which differs across prostheses [3], and requirements of the usual daily-life tasks.

\section{REFERENCES}

[1] C. MacKenzie and T. Iberall, The Grasping Hand. Amsterdam: Elsevier B.V, 2010.

[2] P. Parker, K. Englehart, and B. Hudgins, "Myoelectric signal processing for control of powered limb prostheses.," $J$. Electromyogr. Kinesiol., vol. 16, no. 6, pp. 541-8, Dec. 2006.

[3] J. T. Belter, J. L. Segil, A. M. Dollar, and R. F. Weir, "Mechanical design and performance specifications of anthropomorphic prosthetic hands: a review.," J. Rehabil. Res. Dev., vol. 50, no. 5, pp. 599-618, Jan. 2013.

[4] "Vincent Evolution 2 Hand." [Online]. Available: http://vincentsystems.de/en/prosthetics/vincent-evolution-2/. [Accessed: 09-Oct-2014].

[5] D. S. Childress, "Closed-loop control in prosthetic systems: Historical perspective," Ann. Biomed. Eng., vol. 8, no. 4-6, pp. 293303, Jul. 1980.

[6] S. Raspopovic, M. Capogrosso, F. M. Petrini, M. Bonizzato, J. Rigosa, G. Di Pino, J. Carpaneto, M. Controzzi, T. Boretius, E. Fernandez, G. Granata, C. M. Oddo, L. Citi, A. L. Ciancio, C. Cipriani, M. C. Carrozza, W. Jensen, E. Guglielmelli, T. Stieglitz, P. M. Rossini, and S. Micera, "Restoring natural sensory feedback in real-time bidirectional hand prostheses.," Sci. Transl. Med., vol. 6, no. 222, p. 222ra19, Feb. 2014.

[7] G. A. Tabot, J. F. Dammann, J. A. Berg, F. V Tenore, J. L. Boback,
R. J. Vogelstein, and S. J. Bensmaia, "Restoring the sense of touch with a prosthetic hand through a brain interface.," Proc. Natl. Acad. Sci. U. S. A., vol. 110, no. 45, pp. 18279-84, Nov. 2013.

[8] A. Y. Szeto and F. A. Saunders, "Electrocutaneous stimulation for sensory communication in rehabilitation engineering.," IEEE Trans. Biomed. Eng., vol. 29, no. 4, pp. 300-8, Apr. 1982.

[9] K. A. Kaczmarek, J. G. Webster, P. Bach-y-Rita, and W. J. Tompkins, "Electrotactile and vibrotactile displays for sensory substitution systems.," IEEE Trans. Biomed. Eng., vol. 38, no. 1, pp. 1-16, Jan. 1991.

[10] J. S. Hebert, J. L. Olson, M. J. Morhart, M. R. Dawson, P. D. Marasco, T. A. Kuiken, and K. M. Chan, "Novel Targeted Sensory Reinnervation Technique to Restore Functional Hand Sensation After Transhumeral Amputation.," IEEE Trans. Neural Syst. Rehabil. Eng., vol. 22, no. 4, pp. 765-773, 2014.

[11] T. A. Kuiken, G. Li, B. A. Lock, R. D. Lipschutz, L. A. Miller, K. A. Stubblefield, and K. B. Englehart, "Targeted muscle reinnervation for real-time myoelectric control of multifunction artificial arms.," JAMA, vol. 301, no. 6, pp. 619-28, Feb. 2009.

[12] T. A. Kuiken, G. A. Dumanian, R. D. Lipschutz, L. A. Miller, and K. A. Stubblefield, "The use of targeted muscle reinnervation for improved myoelectric prosthesis control in a bilateral shoulder disarticulation amputee.," Prosthet. Orthot. Int., vol. 28, no. 3, pp. 245-53, Dec. 2004.

[13] S. Dosen, M.-C. Schaeffer, and D. Farina, "Time-division multiplexing for myoelectric closed-loop control using electrotactile feedback.," J. Neuroeng. Rehabil., vol. 11, no. 1, p. 138, Jan. 2014.

[14] C. Hartmann, S. Dosen, S. Amsuess, and D. Farina, "Closed-Loop Control of Myoelectric Prostheses With Electrotactile Feedback: Influence of Stimulation Artifact and Blanking," IEEE Trans. Neural Syst. Rehabil. Eng., vol. 23, no. 5, pp. 807-816, Sep. 2015.

[15] C. Frigo, M. Ferrarin, W. Frasson, E. Pavan, and R. Thorsen, "EMG signals detection and processing for on-line control of functional electrical stimulation.," J. Electromyogr. Kinesiol., vol. 10, no. 5, pp. 351-60, Oct. 2000.

[16] C. Antfolk, M. D’Alonzo, B. Rosén, G. Lundborg, F. Sebelius, and C. Cipriani, "Sensory feedback in upper limb prosthetics.," Expert Rev. Med. Devices, vol. 10, no. 1, pp. 45-54, Jan. 2013.

[17] C. Cipriani, F. Zaccone, S. Micera, and M. C. Carrozza, "On the Shared Control of an EMG-Controlled Prosthetic Hand: Analysis of User-Prosthesis Interaction," IEEE Trans. Robot., vol. 24, no. 1, pp. 170-184, Feb. 2008.

[18] C. Pylatiuk, A. Kargov, and S. Schulz, "Design and Evaluation of a Low-Cost Force Feedback System for Myoelectric Prosthetic Hands," JPO J. Prosthetics Orthot., vol. 18, no. 2, pp. 57-61, Apr. 2006.

[19] C. Cipriani, M. D’Alonzo, and M. C. Carrozza, "A miniature vibrotactile sensory substitution device for multifingered hand prosthetics.," IEEE Trans. Biomed. Eng., vol. 59, no. 2, pp. 400-8, Feb. 2012.

[20] M. Azadi and L. A. Jones, "Vibrotactile actuators: Effect of load and body site on performance," in 2014 IEEE Haptics Symposium (HAPTICS), 2014, pp. 351-356.

[21] L. A. Jones and N. B. Sarter, "Tactile displays: guidance for their design and application.," Hum. Factors, vol. 50, no. 1, pp. 90-111, Feb. 2008.

[22] A. Chatterjee, P. Chaubey, J. Martin, and N. Thakor, "Testing a Prosthetic Haptic Feedback Simulator With an Interactive Force Matching Task," JPO J. Prosthetics Orthot., vol. 20, no. 2, pp. 2734, Apr. 2008.

[23] H. J. Witteveen, H. S. Rietman, and P. H. Veltink, "Vibrotactile grasping force and hand aperture feedback for myoelectric forearm prosthesis users.," Prosthet. Orthot. Int., vol. 39, no. 3, pp. 204-12, Jun. 2015.

[24] R. E. Prior, J. Lyman, P. A. Case, and C. M. Scott, "Supplemental sensory feedback for the VA/NU myoelectric hand. Background and preliminary designs.," Bull. Prosthet. Res., pp. 170-91, Jan. 1976.

[25] N. Jorgovanovic, S. Dosen, D. J. Djozic, G. Krajoski, and D. Farina, "Virtual grasping: closed-loop force control using electrotactile feedback.," Comput. Math. Methods Med., vol. 2014, p. 120357, Jan. 2014.

[26] I. Saunders and S. Vijayakumar, "The role of feed-forward and feedback processes for closed-loop prosthesis control.," $J$. Neuroeng. Rehabil., vol. 8, no. 1, p. 60, Jan. 2011.

[27] H. J. B. Witteveen, E. a Droog, J. S. Rietman, and P. H. Veltink, 
"Vibro- and electrotactile user feedback on hand opening for myoelectric forearm prostheses.," IEEE Trans. Biomed. Eng., vol. 59, no. 8, pp. 2219-26, Aug. 2012.

[28] C. Antfolk and C. Balkenius, "A tactile display system for hand prostheses to discriminate pressure and individual finger localization," J. Med. Biol. Eng., vol. 30, no. 6, pp. 355-360, 2010.

[29] C. Antfolk, M. D’Alonzo, M. Controzzi, G. Lundborg, B. Rosén, F. Sebelius, and C. Cipriani, "Artificial redirection of sensation from prosthetic fingers to the phantom hand map on transradial amputees: vibrotactile versus mechanotactile sensory feedback.," IEEE Trans. Neural Syst. Rehabil. Eng., vol. 21, no. 1, pp. 112-20, Jan. 2013.

[30] M. Solomonow, J. Lyman, and A. Freedy, "Electrotactile two-point discrimination as a function of frequency, body site, laterality, and stimulation codes.," Ann. Biomed. Eng., vol. 5, no. 1, pp. 47-60, Mar. 1977.

[31] S. Dosen, M. Markovic, C. Hartmann, and D. Farina, "Sensory Feedback in Prosthetics: A Standardized Test Bench for ClosedLoop Control," IEEE Trans. Neural Syst. Rehabil. Eng., vol. 23, no. 2, pp. 267-276, Mar. 2015.

[32] F. A. A. Kingdom and N. Prins, Psychophysics: A Practical Introduction. Academic Press, 2009.

[33] A. Ninu, S. Dosen, S. Muceli, F. Rattay, H. Dietl, and D. Farina, "Closed-Loop Control of Grasping With a Myoelectric Hand Prosthesis: Which Are the Relevant Feedback Variables for Force Control?," IEEE Trans. Neural Syst. Rehabil. Eng., vol. 22, no. 5, pp. 1041-1052, Sep. 2014.

[34] S. Dosen, M. Markovic, N. Wille, M. Henkel, M. Koppe, A. Ninu, C. Frömmel, and D. Farina, "Building an internal model of a myoelectric prosthesis via closed-loop control for consistent and routine grasping," Exp. Brain Res., vol. 233, no. 6, pp. 1855-1865, Jun. 2015.

[35] M. D'Alonzo, S. Dosen, C. Cipriani, and D. Farina, "HyVE: Hybrid Vibro-Electrotactile Stimulation for Sensory Feedback and Substitution in Rehabilitation," IEEE Trans. Neural Syst. Rehabil.
Eng., vol. 22, no. 2, pp. 290-301, Mar. 2014.

[36] R. E. Johnson, K. P. Kording, L. J. Hargrove, and J. W. Sensinger, "Does EMG control lead to distinct motor adaptation?," Front. Neurosci., vol. 8, p. 302, Jan. 2014.

[37] K. Wei and K. Körding, "Uncertainty of feedback and state estimation determines the speed of motor adaptation.," Front. Comput. Neurosci., vol. 4, p. 11, Jan. 2010.

[38] D. W. Franklin and D. M. Wolpert, "Computational mechanisms of sensorimotor control.," Neuron, vol. 72, no. 3, pp. 425-42, Nov. 2011.

[39] E. Todorov, "Optimality principles in sensorimotor control.," Nat. Neurosci., vol. 7, no. 9, pp. 907-15, Sep. 2004.

[40] P. F. Pasquina, M. Evangelista, A. J. Carvalho, J. Lockhart, S. Griffin, G. Nanos, P. McKay, M. Hansen, D. Ipsen, J. Vandersea, J. Butkus, M. Miller, I. Murphy, and D. Hankin, "First-in-man demonstration of a fully implanted myoelectric sensors system to control an advanced electromechanical prosthetic hand.," $J$. Neurosci. Methods, pp. 1-9, Aug. 2014.

[41] J. J. Baker, E. Scheme, K. Englehart, D. T. Hutchinson, and B. Greger, "Continuous detection and decoding of dexterous finger flexions with implantable myoelectric sensors.," IEEE Trans. Neural Syst. Rehabil. Eng., vol. 18, no. 4, pp. 424-32, Aug. 2010.

[42] J. D. Brown, A. Paek, M. Syed, M. K. O’Malley, P. A. Shewokis, J. L. Contreras-Vidal, A. J. Davis, and R. B. Gillespie, "An exploration of grip force regulation with a low-impedance myoelectric prosthesis featuring referred haptic feedback.," $J$. Neuroeng. Rehabil., vol. 12, p. 104, Jan. 2015.

[43] H. Bouwsema, P. J. Kyberd, W. Hill, C. K. van der Sluis, and R. M. Bongers, "Determining skill level in myoelectric prosthesis use with multiple outcome measures.," J. Rehabil. Res. Dev., vol. 49, no. 9, pp. 1331-48, Jan. 2012. 